\title{
Efecto de la exposición a ruido en entornos laborales sobre la calidad de vida y rendimiento
}

\section{Effects of noise exposure in working places on quality of life and performance}

\author{
Elena Ordaz Castillo \\ Escuela Nacional de Medicina del Trabajo \\ Instituto de Salud Carlos III. Madrid. España. \\ Jerónimo Maqueda Blasco \\ Escuela Nacional de Medicina del Trabajo \\ Instituto de Salud Carlos III. Madrid. España.
}

\section{Angel Asúnsolo Del Barco}

Dpto. de Ciencias Sanitarias y Médico Sociales, Universidad de Alcalá de Henares. (Madrid) España.

\section{Agustín Silva Mato}

Dpto. de Ciencias Sanitarias y Médico Sociales, Universidad de Alcalá de Henares. (Madrid) España.

\section{María Fe Gamo González}

Escuela Nacional de Medicina del Trabajo Instituto de Salud Carlos III. Madrid. España.

Rosa Ana Cortés Barragán

Escuela Nacional de Medicina del Trabajo

Instituto de Salud Carlos III. Madrid. España.

\section{Eva Bermejo García}

Escuela Nacional de Medicina del Trabajo

Instituto de Salud Carlos III. Madrid. España.

\section{Correspondencia:}

Jerónimo Maqueda Blasco

Pabellón 8-Ciudad Universitaria.

Madrid 28040

Telef: $91-8224013$

e-mail. jmaqueda@isciii.es

Financiación: FIS PI 07/90034

Resumen

Objetivos: La exposición laboral a ruido origina efectos sobre la salud de carácter no auditivo. El objetivo de este estudio es estimar la asociación entre exposición a ruido en entornos laborales y la aparición de alteraciones en la conducta, rendimiento y síntomas psicosomáticos.

Metodología: Estudio de prevalencia a partir de datos de la VI Encuesta Nacional de Condiciones de Trabajo analizando como variable independiente la exposición a ruido y como variables dependientes los "síntomas que manifiesta el trabajador" tratados de forma individual o por grupo de síntomas según su naturaleza: conductual, psicosomáticos y rendimiento.

La asociación entre exposición y efecto se analizó mediante la prueba de $\mathrm{X}^{2}$ y se calculó el OR de prevalencias ajustado por ritmo de trabajo (IC95\%) para medir la asociación entre "exposición a ruido" y 
grupos de síntomas de forma cruda en el conjunto de la población y de forma estratificada por ramas de actividad.

Resultados: El 36,5\% de la población trabajadora está expuesta a ruido en su puesto de trabajo. Se observa una mayor prevalencia de los grupos de síntomas conductuales, psicosomáticos y de rendimiento $(\mathrm{p}<0,001)$ entre los trabajadores expuestos a ruido frente a no expuestos. La rama de Actividades Sanitarias presenta los mayores valores de OR para el grupo de síntomas conductuales $(\mathrm{OR}=2,53[1,71-3,75])$ y de rendimiento $(\mathrm{OR}=2,26,[1,43-3,56])$ y la Industria Química presenta el mayor grado de asociación entre exposición a ruido y síntomas psicosomáticos (OR=5,37, [1,13-25,55]).

Conclusiones: Se observa una asociación estadísticamente significativa entre exposición a ruido y alteraciones de conducta, rendimiento y síntomas psicosomáticos en la población trabajadora española.

(Med Segur Trab (Internet) 2009; 55 (216): 35-45)

Palabras clave: Ruido en el Ambiente de Trabajo, Evaluación del Rendimiento de Empleados, Conducta, Trastornos Psicofisiológicos, Calidad de vida.

Abstract

Aims: Occupational noise exposure could produce extra-auditory effects. The purpose of this study was to investigate whether occupational noise exposure can influence human behaviour, performance and development of psychosomatic symptoms.

Methodology: Cross-sectional study, from the Data-base of Spanish VI National Survey on Working Conditions, "noise exposure" has been analyzed as independent variable and: "symptoms self-reportered by workers" as effect variable, considering them independently or grouped variables in behavioural, psychosomatic and performance symptoms.

The relationship between exposure and effect was analyzed by X2 test. Prevalence adjusted Odds Ratio by work rhythm (95\% CI), were estimated to measure the association between perceived noise annoyance and grouped symptoms in the population and in the different branches of activity.

Results: $36.5 \%$ of the working population is exposed to noise in their workplace. There is a higher prevalence of behavioural symptoms group, psychosomatic and performance $(\mathrm{p}<0.001)$ among workers exposed to noise compared to unexposed.

Health Care Sector reported the highest risk for behavioural (OR=2,53 [1,71-3,75]) and performance symptoms $(\mathrm{OR}=2,26,[1,43-3,56])$. Chemical Industry had a prevalence ratio between noise and psychosomatic symptoms of $(\mathrm{OR}=5,37 \quad[1,13-25,55])$.

Conclusions: Our results found and statistic significant association between noise annoyance exposure and behavioural, performance and development of psychosomatic symptoms in the Spanish working population.

(Med Segur Trab (Internet) 2009; 55 (216): 35-45)

Key words: Noise, Occupational; Employee Performance Appraisal, behavior, Psychophysiologic Disorders, Quality of Life, work rhythm. 


\section{INTRODUCCIÓN}

Según un informe de la OMS del año 2002 el ruido se incluye dentro de los cinco principales factores de riesgo para la salud en el medio laboral ${ }^{1}$.

Los resultados de la última Encuesta Europea de Condiciones de Trabajo realizada por la Fundación Europea para la Mejora de las Condiciones de Vida y de Trabajo ${ }^{2}$ indican que el $30,2 \%$ de los trabajadores manifestan estar expuestos a altos niveles de ruido durante al menos una cuarta parte de su jornada.

En nuestro país, la VI-Encuesta Nacional de Condiciones de trabajo, fuente de datos nuestro estudio, revela que un $37,2 \%$ de los trabajadores perciben el ruido en su puesto de trabajo como molesto o muy elevado ${ }^{3}$.

El principal efecto de la exposición prolongada al ruido en la salud de los trabajadores es la pérdida auditiva (hipoacusia, sordera profesional) que es, sin duda, el efecto más documentado y más frecuentemente reconocido como Enfermedad Profesional (EP) en los Estados Miembros de la UE. ${ }^{4-6}$. En España en el año 2008 se notificaron a la Seguridad Social 790 casos de hipoacusia/sordera de un total de 18.700 casos de EP (4,2\% del total) $)^{7}$.

La legislación vigente sobre protección de la salud y la seguridad de los trabajadores contra los riesgos relacionados con la exposición al ruido establece valores límite de exposición dirigidos fundamentalmente a prevenir la hipoacusia o sordera profesional ${ }^{8}$. No obstante se han documentado otros efectos sobre la salud por exposición a ruido de naturaleza extra-auditiva que, en algunos casos, se producen por debajo de estos valores.

Este hecho se pone de manifiesto en las dos revisiones sistemáticas llevadas a cabo por la "Health and Safety Executive." en Reino Unido,10, si bien las limitaciones metodológicas de muchos de los estudios y los resultados contrapuestos no permiten realizar afirmaciones concluyentes al respecto.

En general, estos efectos extra-auditivos están mediados por una reacción de estrés frente al ruido que desencadena en el organismo una respuesta, como lo haría ante cualquier agresión de tipo físico o psíquico ${ }^{11}$. Entre estos efectos figuran las enfermedades cardiovasculares ${ }^{12-14}$, efectos sobre el sueño y la fatiga ${ }^{15-17}$, alteraciones sobre la reproducción y el desarrollo ${ }^{18,19}$, alteraciones psicosociales y psicológicas ${ }^{16,20,21}$, entre otros muchos.

Todos estos efectos tienen una repercusión directa sobre la calidad de vida, entendida ésta como la sensación de bienestar del individuo con el medio que le rodea, perturbando así el desarrollo personal, familiar y laboral. En este sentido, el Tribunal Constitucional, refiriéndose a una demanda por ruido ambiental en el año 2004, falló qué "el ruido, en la sociedad de nuestros días, puede llegar a representar un factor psicopatógeno y una fuente permanente de perturbación de la calidad de vida de los ciudadanos"22.

Entre los distintos y numerosos instrumentos existentes para evaluar la calidad de vida, todos ellos coinciden en considerar variables relacionadas con la salud física, el control emocional o conductual y las alteraciones de origen psicológicos tales como la ansiedad o la depresión ${ }^{23-25}$.

Por otro lado, distintos autores han demostrado que el ruido, a una intensidad moderada, puede influir en el rendimiento de los trabajadores ${ }^{26,27}$. El grado de interferencia dependerá de la naturaleza del ruido, del tipo de tarea y también de la susceptibilidad del individuo.

El ruido no es el único factor fuente de estrés al que está sometido el trabajador. Un ritmo de trabajo excesivo puede ocasionar alteraciones de naturaleza física y psíquica e influir por tanto en los efectos del ruido sobre la salud. 


\section{OBJETIVOS}

Considerando los antecedentes analizados anteriormente, el presente estudio persigue como objetivo estimar la asociación entre percepción molesta a ruido y alteraciones en conducta, rendimiento y síntomas psicosomáticos en entornos laborales valorando si el ritmo de trabajo influye en esta asociación.

\section{MATERIAL Y MÉTODOS}

Estudio de prevalencia a partir de los datos de la VI Encuesta Nacional de Condiciones de Trabajo (VI-ENCT) del Instituto Nacional de Seguridad e Higiene en el Trabajo del Ministerio de Trabajo e Inmigración sobre una muestra de 11.054 entrevistas a trabajadores con un error muestral para un nivel de confianza del $95,5 \%$ y $p=q$ de $\pm 0,95 \%$.

Como variable de exposición se generó una variable dicotómica de "expuestos /no expuestos a ruido" a partir de la variable original del cuestionario de la VI-ENCT que recoge el nivel percibido de ruido en el puesto de trabajo como una variable categórica ordinal con cuatro categorías: "muy bajo", "molesto", "elevado" y "muy elevado" .

Esta nueva variable dicotómica considera "expuestos a ruido" a aquellos trabajadores en cuyo puesto de trabajo el nivel de ruido es percibido como: molesto, elevado o muy elevado y "no expuestos a ruido" a los trabajadores que perciben que en su puesto de trabajo el nivel de ruido es muy bajo o casi no hay ruido.

Como variables de efecto se han analizado 12 variables de respuesta múltiple transformadas en dicotómicas sobre presencia de determinados síntomas, pertenecientes a la pregunta 66 del cuestionario del trabajador de la VI- ENCT. Así mismo, se han creado tres nuevas variables dicotómicas a partir de la agrupación de las 12 variables anteriores según la naturaleza del síntoma en alteraciones de la Conducta, del Rendimiento o Psicosomáticas. (Tabla 1).

Tabla 1. Variables de Efecto

\begin{tabular}{|c|c|c|c|}
\hline \multicolumn{4}{|c|}{ ALTERACIONES } \\
\hline & CONDUCTA & RENDIMIENTO & PSICOSOMÁTICAS \\
\hline \multirow{5}{*}{ Síntomas } & Dormir mal & $\begin{array}{l}\text { Sensación continua de } \\
\text { cansancio }\end{array}$ & Dolores de cabeza \\
\hline & Irritabilidad & Dificultad de concentración & Problemas oculares \\
\hline & Agotamiento emocional & Alteraciones de la memoria & $\begin{array}{c}\text { Alteraciones del Apetito } \\
\text { o Digestivas }\end{array}$ \\
\hline & $\begin{array}{c}\text { No olvida los problemas } \\
\text { del trabajo }\end{array}$ & & Mareos \\
\hline & Bajo estado de ánimo & & \\
\hline
\end{tabular}

La asociación entre grupos de trabajadores expuestos y no expuestos a ruido para las distintas variables de efecto estudiadas se analizó mediante $\mathrm{X}^{2}$.

En la estimación de la asociación entre exposición molesta a ruido y las tres agrupaciones de síntomas en el total de la población y tras estratificar por rama de actividad se emplearon modelos de Regresión Logística para el cálculo de la OR de prevalencias.

Para identificar la influencia del factor ritmo de trabajo sobre el grupo de síntomas relacionados con la conducta, rendimiento y alteraciones psicosomáticas, se controló el efecto de la exposición por la variable dicotómica "expuestos a ritmo de trabajo rápido". 
Esta variable se creó a partir de la variable "trabajar muy rápido" (pregunta p32-2 del cuestionario de la VI-ENCT), recogida en 5 categorías ("siempre o casi siempre", " $a$ menudo", "a veces", "raramente" y "casi nunca o nunca"), considerando "expuestos a ritmo de trabajo rápido" aquellos que respondieron la opción siempre o casi siempre y como "no expuestos a ritmo de trabajo rápido" el resto de categorías.

El programa estadístico utilizado ha sido el SPSS versión 16.0.

\section{RESULTADOS}

El 36,5\% de los trabajadores entrevistados en la VI-ENCT están expuestos a ruido en su puesto de trabajo (Figura 1).

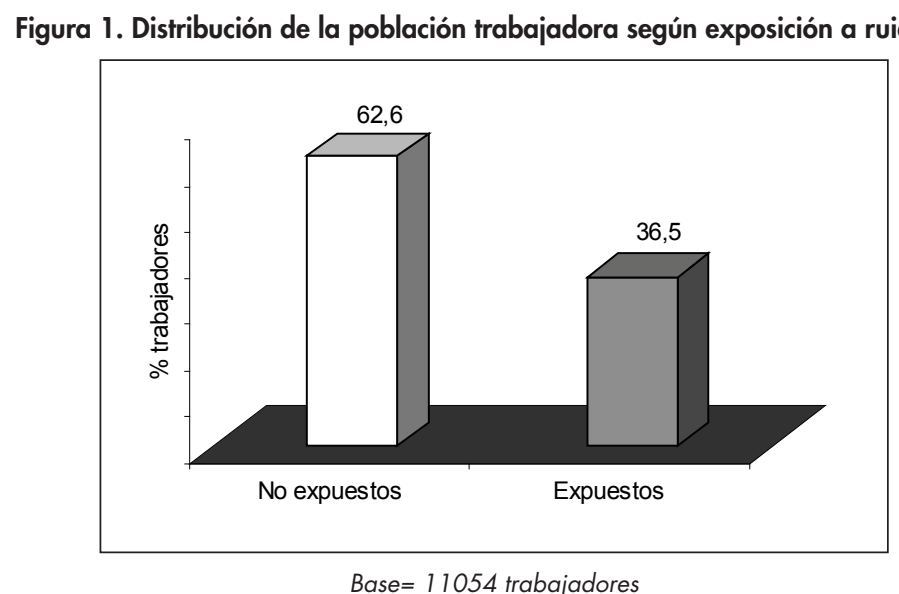

La prevalencia de las tres agrupaciones de síntomas, conductuales, psicosomáticos y relacionados con el rendimiento, es estadísticamente $(\mathrm{p}<0,001)$ mayor en el grupo de trabajadores expuestos a ruido (Figura 2).

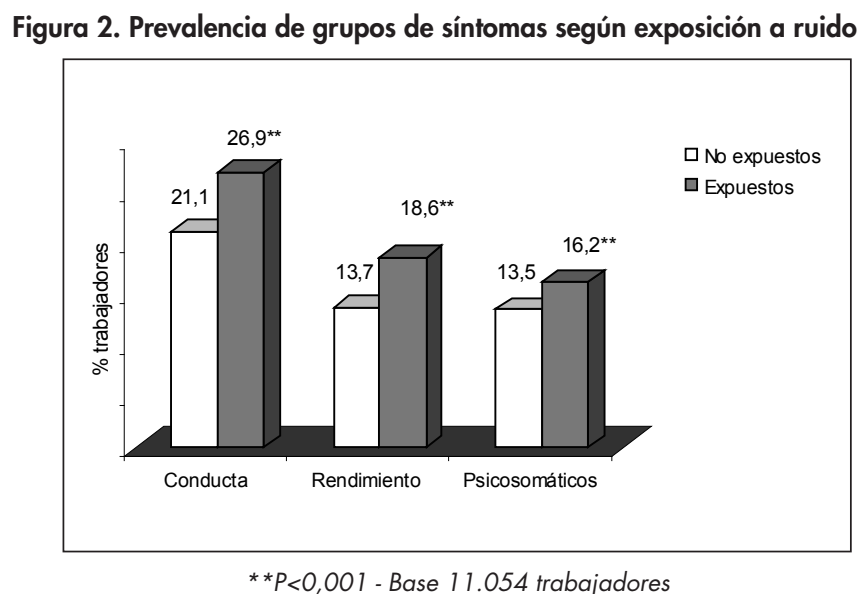

Dentro del grupo de "alteraciones conductuales" el síntoma más prevalente fue dormir mal (14,6\% vs 10,8\%); en el grupo de "alteraciones del rendimiento" destacó la sensación continúa de cansancio (15,5\% vs 10,6\%) y en el grupo de "síntomas psicosomáticos" sufrir dolores de cabeza (12\% vs 10,1\%). (Figura 3).

Figura 3. Prevalencias, expresadas en $\%$, de síntomas según grupo y nivel percibido de ruido 


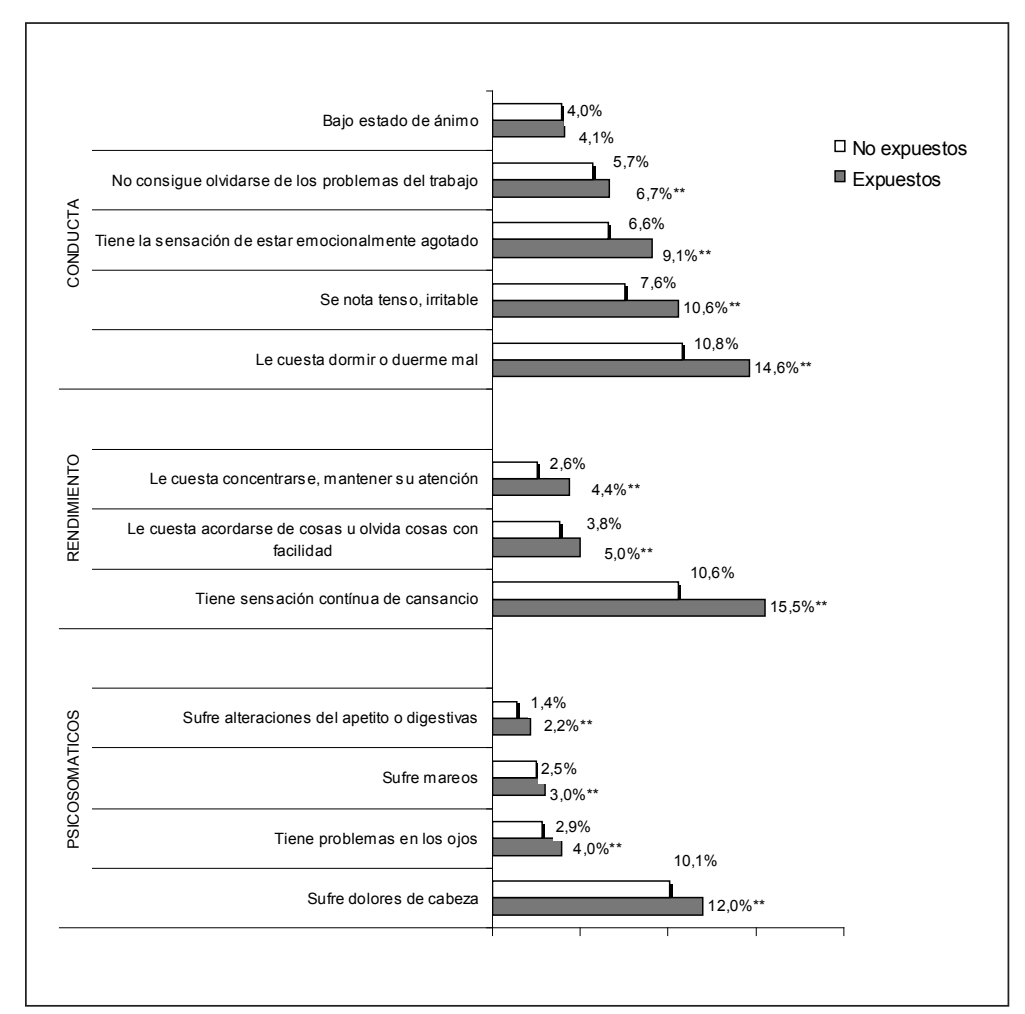

Base 11.054 trabajadores $-P<0,001$

El mayor riesgo entre exposición profesional a ruido y los tres grupos de efecto, una vez controlado el efecto del ritmo de trabajo $\left(\mathrm{OR}_{\mathrm{a}}\right)$, se obtuvo para la variable "alteraciones del rendimiento" $\mathrm{OR}_{\mathrm{a}}=1,33$ [IC95\% 1,19-1,47] (Tabla 2).

Tabla 2. Asociación entre exposición a ruido laboral y grupos de síntomas

\begin{tabular}{lcccc}
\hline & OR & IC95\% & OR $_{\mathbf{a}}$ & IC $_{\mathbf{a}}$ 95\% \\
\hline Rendimiento & 1,43 & $1,29-1,59$ & 1,33 & $1,19-1,47$ \\
Conducta & 1,37 & $1,25-1,50$ & 1,27 & $1,16-1,40$ \\
Psicosomático & 1,24 & $1,10-14$ & 1,15 & $1,03-1,28$
\end{tabular}

OR (Odss Ratio cruda)

$\mathrm{OR}_{\mathrm{a}}$ (Odss Ratio ajustada por ritmo de trabajo)

La tabla 3 resume los valores de OR (crudo y ajustado por ritmo de trabajo) por rama de actividad entre exposición a ruido y cada grupo de síntomas. 


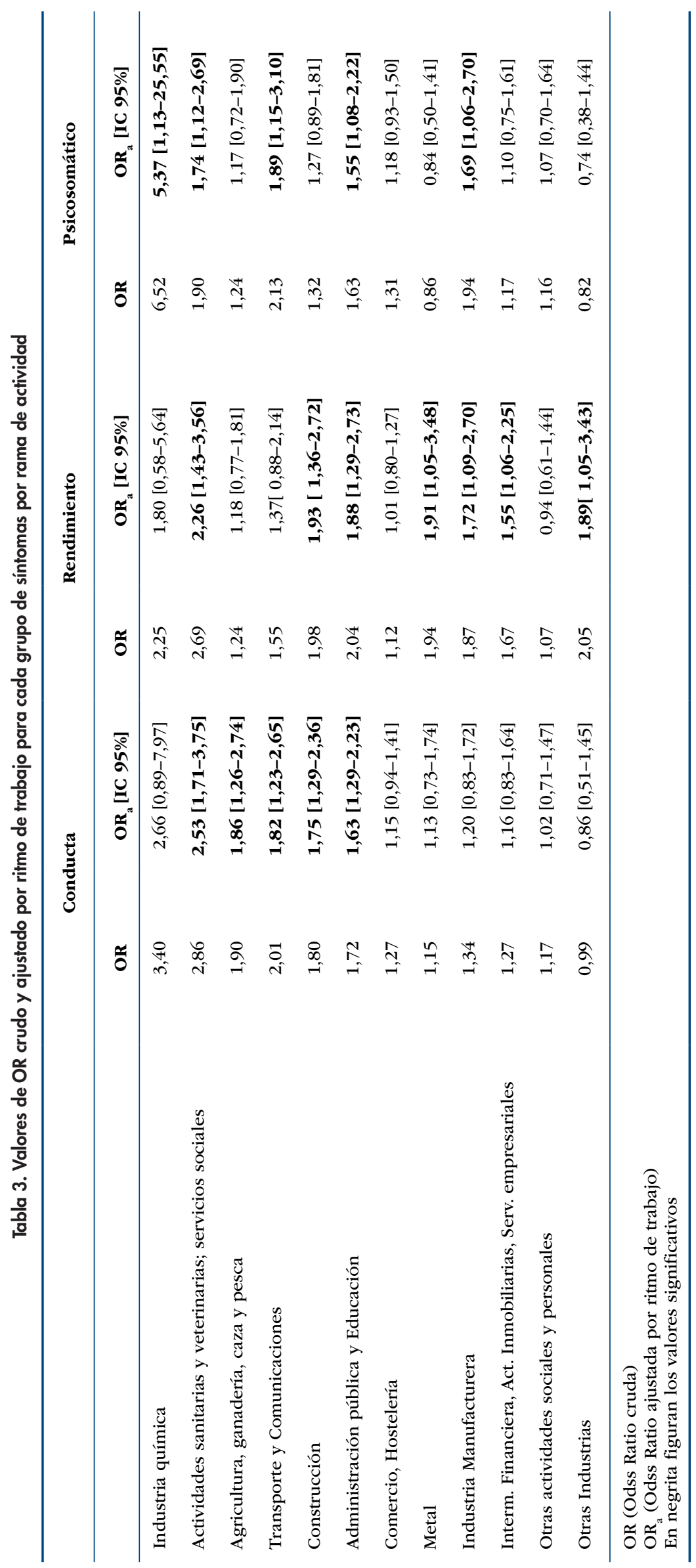


De las 12 ramas de actividad estudiadas y una vez controlado por ritmo de trabajo, el "rendimiento" fue el grupo de alteraciones que se asoció, de forma significativa a la exposición al ruido, en un mayor número de actividades", seguido de las "alteraciones de conducta" $\mathrm{y}$ "psicosomáticas".

El grado de asociación para el grupo de "alteraciones de conducta" fue mayor en la rama de Actividades Sanitarias y Veterinarias $\mathrm{OR}_{\mathrm{a}}=2,53$ [1,71-3,74]. En cuanto a las "alteraciones del rendimiento", el mayor grado de asociación positiva y significativa con la exposición a ruido se dio en la rama de Actividades Sanitarias y Veterinarias $\mathrm{OR}_{\mathrm{a}}=2,26$ [1,43-3,56]. Finalmente, la mayor asociación entre el grupo de "síntomas psicosomáticos" y exposición a ruido, ajustado por ritmo de trabajo, fue con la rama de la Industria Química OR =5,37 [1,13-25,5].

\section{DISCUSIÓN}

Los datos de la VI-ENCT reflejan que el 36,5\% de la población trabajadora española percibe el ruido en su puesto de trabajo entre molesto y muy elevado, percepción que se relaciona de forma estadísticamente significativa con la presencia de alteraciones en el rendimiento del trabajador así como con alteraciones de carácter conductual y psicosomáticas lo que repercute, en la calidad de vida del trabajador.

El perfil de morbilidad de la población trabajadora expuesta ruido se caracteriza principalmente por dormir mal, tener sensación continua de cansancio y sufrir dolores de cabeza.

La asociación encontrada en nuestros resultados entre exposición a ruido molesto y "alteraciones en el rendimiento" de los trabajadores coincide con resultados de estudios previos donde el rendimiento expresado en función de variables tales como control de la actividad, rapidez de reacción, aprendizaje, memorización o inteligencia también se veía mermado por la exposición a ambientes ruidosos ${ }^{27,28,29}$.

Del mismo modo, la asociación entre exposición a ruido y "alteraciones en la conducta" definida en nuestro estudio por variables como: dormir mal, irritabilidad, agotamiento emocional, bajo estado de ánimo y la dificultad para olvidarse de los problemas de trabajo, es coincidente con lo resultados de otros estudios. Gitanjali B. \& Dhamotharan R. ${ }^{15}$ observaron, en un estudio de cohorte retrospectivo, que trabajadores expuestos a ruido presentaban una pobre calidad de sueño; Saremi, $M \& \mathrm{col}^{130}$ sugirieron que el factor principal de fatiga relacionada con el trabajo podría ser la exposición a ruido ocupacional y Melamed S. \& col $^{31}$ destacaron el peso del ruido sobre la carga de trabajo, el malestar y la irritabilidad post-laboral en un estudio que medía el efecto combinado del ruido y una elevada complejidad laboral.

Por otro lado, las "alteraciones psicosomáticas" por exposición a ruido encontradas en este estudio tales como las cefaleas, mareos, problemas oculares o digestivos, si bien han resultado menos prevalentes y no tan documentadas previamente, son puesta de manifiesto por otros autores ${ }^{32}$.

Del análisis estratificado por ramas de actividad se desprende que Actividades Sanitarias y Veterinarias, Administración Pública, Transporte y Comunicaciones, Construcción e Industrias manufactureras constituyen las ramas de interés preferente para la instauración de programas de control del ruido que aborden efectos de naturaleza extra-auditiva. 
En resumen, por las implicaciones de las alteraciones conductuales y psicosomáticas en la calidad de vida, podemos concluir que la exposición laboral a ruido percibido como molesto, elevado y muy elevado, disminuye la calidad de vida de los trabajadores en general, así como su rendimiento laboral potenciando efectos tales como la sensación continúa de cansancio, la falta de concentración y de memoria.

\section{AGRADECIMIENTOS}

A María Felix Ortíz Jiménez por su amable colaboración. 


\section{BIBLIOGRAFIA}

1. Worlh Health Organization. The word health report 2002: Reducing risk, promoting healthy life. Worlh Health Organization, 2002. Disponible en: www.who.int/whr/2002/

2. Parent-Thirion A, Fernández Macías E, Hurley J, Vermeylen G. Fourth European Working Conditions Survey. Disponible en: www.eurofound.europa.eu/publications/htmlfiles/ef0698.htm

3. Instituto de Seguridad e higiene en el Trabajo. VI Encuesta Nacional de Condiciones de Trabajo. Ministerio de Trabajo y Asuntos sociales, 2004.Disponible en:www.oect.es/portal/site/Observatorio/menuitem.88888a 9742719027d4617410060961ca/?vgnextoid=cc8f6a5f01d63110VgnVCM100000dc0ca8c0RCRD

4. Ogido R, Costa EA, Machado HC. Prevalence of auditory and vestibular symptoms among workers exposed to occupational noise] Rev Saude Publica 2009; 43(2):377-380.

5. Tunay M, Melemez K. Noise induced hearing loss of forest workers in Turkey. Pak J Biol Sci 2008; 11(17):2144-2148.

6. Liu YM, Li XD, Li YS, Guo X, Xiao LW, Xiao QH et al. [Effect of environmental risk factors in occupational noise exposure to Noise-induced hearing loss]. Zhonghua Lao Dong Wei Sheng Zhi Ye Bing Za Zhi 2008; 26(12):721-724.

7. Seguridad Social. Observatorio de Enfermedades Profesionales. $\mathrm{N}^{\mathrm{o}}$ de partes comunicados por grupos de enfermedad y agente causante. Ministerio de Trabajo e Inmigración. Disponible en: www.seg-social.es/ Internet_1/Estadistica/Est/Observatorio_de_las_Enfermedades_Profesionales/index.htm

8. Boletín Oficial del Estado. Real Decreto 286/2006, de 10 de marzo, sobre la protección de la salud y la seguridad de los trabajadores contra los riesgos relacionados con la exposición al ruido. BOE núm. 60 de 11/03/2009.

9. B Butler M, Graveling R, Pilkington A, Boyle AL. Non-auditory effects of noise at work: A critical review of the literature post 1988. Institute of Occupational Medicine. Health and Safety Executive. Disponible en : www.hse.gov.uk/research/crr_pdf/1999/crr99241.pdf

10. Smith A, Broadbent D. Non-auditory effects of noise at work a review of the literature. Health and Safety Executive HSE, 1991. Disponible en :www.hse.gov.uk/research/crr_pdf/1991/crr91030.pdf

11. Babisch W.T. The Noise/stress concept, risk assessment and research needs. Noise Health 2002; 4:210-216.

12. Babisch W, Beule B, Schust M, Kersten N, Ising H. Traffic noise and risk of myocardial infarction. Epidemiology $2005 ; 16(1): 33-40$.

13. Babisch W. Road traffic noise and cardiovascular risk. Noise Health 2008; 10(38):27-33.

14. Chang TY, Jain RM, Wang CS, Chan CC. Effects of occupational noise exposure on blood pressure. J Occup Environ Med 2003; 45(12):1289-1296.

15. Gitanjali B, Dhamotharan R. Effect of occupational noise on the nocturnal sleep architecture of healthy subjects. Indian J Physiol Pharmacol 2003; 47(4):415-422.

16. Leventhall HG. Low frequency noise and annoyance. Noise Health 2004; 6(23):59-72.

17. Rios AL, da Silva GA. Sleep quality in noise exposed Brazilian workers. Noise Health 2005; 7(29):1-6.

18. Gerhardt KJ, Abrams RM. Fetal exposures to sound and vibroacoustic stimulation. J Perinatol 2000; 20(8 Pt 2):S21-S30.

19. Kawada T. The effect of noise on the health of children. Noise: a hazard for the fetus and newborn. American Academy of Pediatrics. Committee on Environmental Health. J Nippon Med Sch 2004; 71(1):5-10.

20. Cohen S. After effects of stress on human performance and social behavior: a review of research and theory. Psychol Bull 1980; 88(1):82-108.

21. Morrison WE, Haas EC, Shaffner DH, Garrett ES, Fackler JC. Noise, stress, and annoyance in a pediatric intensive care unit. Crit Care Med 2003; 31(1):113-119.

22. Tribunal Constitucional. Sentencia 16/2004 de 23 de Febrero. $N^{o}$ de recurso: 1784/1999 Derecho a la integridad física $\mathrm{y}$ moral, $\mathrm{y}$ derecho a la intimidad personal $\mathrm{y}$ familiar, posiblemente vulnerados por exposición prolongada a niveles de ruido evitables e insoportables. Disponilbe en : www.ruidos.org/ Jurisprudencia/TC_040223.html

23. García-Riaño D IE. Calidad de Vida en Enfermos Físicos. Rev Psiquiatría 1992; 19(4):148-161.

24. Ware JE SCD. The MOS 36-item short-form health survey (SF-36). Conceptual framework and item selection. Medical Care 1992; 30:473-483.

25. The World Health Organization. WHOQOL.: Measuring of Quality of Life World Health World Health Organization. Disponible en :www.who.int/entity/mental_health/media/68.pdf

26. Muzammil M, Khan AA, Hasan F, Hasan SN. Effect of noise on human performance under variable load in a die casting industry--a case study. J Environ Sci Eng 2004; 46(1):49-54. 
27. Pawlaczyk-Luszczyniska M, Dudarewicz A, Waszkowska M, Szymczak W, Sliwinska-Kowalska M. The impact of low-frequency noise on human mental performance. Int J Occup Med Environ Health 2005; 18(2):185-198.

28. Melamed S, Bruhis S. The effects of chronic industrial noise exposure on urinary cortisol, fatigue and irritability: a controlled field experiment. J Occup Environ Med 1996; 38(3):252-256.

29. Waye KP, Bengtsson J, Rylander R, Hucklebridge F, Evans P, Clow A. Low frequency noise enhances cortisol among noise sensitive subjects during work performance. Life Sci 2002; 70(7):745-758.

30. Saremi M, Rohmer O, Burgmeier A, Bonnefond A, Muzet A, Tassi P. Combined effects of noise and shift work on fatigue as a function of age. Int J Occup Saf Ergon 2008; 14(4):387-394.

31. Melamed S, Fried Y, Froom P. The joint effect of noise exposure and job complexity on distress and injury risk among men and women: the cardiovascular occupational risk factors determination in Israel study. J Occup Environ Med 2004; 46(10):1023-1032.

32. Kryter KD. The Effects of Noise on Man. second edition, Academic Press ed. London: 1985. 